\title{
JUAN ANTONIO FERNÁNDEZ (1752-1814), EL EPIGRAFISTA ACCIDENTAL
}

\author{
Juan Manuel Abascal Palazón \\ Universidad de Alicante \\ juan.abascal@ua.es
}

\section{JUAN ANTONIO FERNÁNDEZ (1752-1814), THE ACCIDENTAL EPIGRAPHER}

\begin{abstract}
RESUMEN:En 1789, a instancias del prior Antonio Tavira, Juan Antonio Fernández se trasladó a Uclés (Cuenca) para llevar a cabo la ordenación y clasificación del archivo de la Orden de Santiago. Allí supo de la existencia de las ruinas romanas de Cabeza del Griego (Segobriga) y llevó a cabo con Tavira las primeras excavaciones. Durante ese tiempo, descubrieron un buen número de inscripciones romanas y Juan Antonio Fernández llevó a cabo la primera recopilación de las inscripciones de Segobriga. Después de su muerte en 1814, muchos de los documentos fueron comprados por la Real Academia de la Historia.

PALABRAS CLAVE: Uclés, Segobriga, Antonio Tavira, inscripciones romanas
\end{abstract}

\begin{abstract}
In 1789, at the request of the prior Antonio Tavira, Juan Antonio Fernández moved to Uclés (Cuenca) to carry out the management of the main archive of the Order of Santiago. There he learned of the existence of the Roman ruins at the near spot of Cabeza del Griego (Segobriga) and undertook the first archaeological excavations with Tavira. In that time, were discovered many Roman inscriptions and Juan Antonio Fernández carried out the first compilation of the inscriptions of Segobriga. After his death in 1814, many of its documents were bought by the Royal Academy of history.
\end{abstract}

KEYWORDS: Uclés, Segobriga, Antonio Tavira, Roman inscriptions

RECIBIDO: 10.03.2014. ACEPTADO: 27.05.2014

Después de haber sido la sede del archivo de la Orden de Santiago durante varios siglos, el monasterio de Uclés (Cuenca) perdió esa condición con la supresión por Real Decreto de 9 de marzo de 1836 de las casas matrices de las cuatro Órdenes Militares españolas ${ }^{1}$. Atrás quedaban los tiempos de gloria en que el conventual

${ }^{1}$ Este trabajo se ha escrito en el marco del proyecto HAR2012-32881 ("Manuscritos epigráficos españoles de los siglos XVI-XIX: entre la historiografía y la epigrafía científica"), financiado por el Gobierno de España. En todos los casos, las siglas RAH aluden al archivo de la Real Academia de la Historia. 
fue visitado por reyes, viajeros ilustres, historiadores y cronistas, que bien recalaban en su refectorio en los trayectos que desde Madrid llevaban a las ciudades de la costa mediterránea o bien acudían a la consulta de su nutrido y valioso archivo, parte del cual se perdió durante la Guerra de la Independencia antes de ser trasladado en 1872 al Archivo Histórico Nacional, en donde hoy se conserva.

En 1574, Ambrosio de Morales acudió a Uclés para revisar ese archivo santiaguista y extractó un buen número de documentos en un manuscrito que contiene sólo una acumulación de datos y que editaría en 1793 el impresor madrileño Benito $\mathrm{Cano}^{2}$. Al comentar ese manuscrito por encargo del editor, Juan Antonio Fernández -el personaje objeto de estas páginas y al que ahora presentaremosescribió lo siguiente:

Si Morales hubiera tenido la felicidad de hallar libre de la confusión, que en su tiempo padecía, el mencionado archivo, era regular que nos hubiese dado noticia de todos los privilegios y preciosos documentos que encierra ${ }^{3}$.

Fernández sabía perfectamente de lo que hablaba y era la persona adecuada para comentar las notas de Morales, puesto que durante los cuatro últimos años había ostentado la condición de archivero del citado monasterio y el 14 de mayo de 1793 se había convertido por designación real en Archivero general de la Orden de Santiago ${ }^{4}$.

Juan Antonio Fernández Pascual nació el 29 de marzo de 1752 en Tudela, ciudad en la que luego fijó su residencia. Por sus escritos, su correspondencia y los estudios de quienes se han ocupado de su perfil biográfico ${ }^{5}$, sabemos de su

\footnotetext{
${ }^{2}$ A. de Morales, "Noticias históricas sacadas del archivo de Uclés, de sus sepulcros y calenda, y del testamento del infante Don Enrique, con un cronicón hasta ahora no publicado", Opúsculos castellanos de Ambrosio de Morales, cuyos originales se conservan inéditos en la Real biblioteca del monasterio del Escorial, Madrid, en la oficina de D. Benito Cano, 1793, vol. 2, 7-54.

${ }^{3}$ J. A. Fernández, Carta a Benito Cano, sin fecha [1793], editada como preámbulo al texto de A. de Morales, "Noticias históricas ..." (op. cit. en nota 2) 4.

${ }^{4}$ J. R. Castro Álava, "Figuras tudelanas: Juan Antonio Fernández, archivero de la Orden de Santiago", Príncipe de Viana 2.2 (1941) 112-113.

${ }^{5}$ La figura de Juan Antonio Fernández ha sido tratada en diferentes ocasiones, por lo que prescindimos de los aspectos biográficos y de las noticias sobre su entorno familiar, que pueden verse en las siguientes obras: M. Sáinz y Pérez de Laborda, Apuntes tudelanos (Tudela 1913) 388-401; J. R. Castro Álava, "Figuras tudelanas: Juan Antonio Fernández, archivero de la Orden de Santiago", Príncipe de Viana 2.2 (1941) 95-122; F. Fuentes Pascual, "Más notas sobre Juan Antonio Fernández", Príncipe de Viana 16.59 (1955) 203-231; J. R. Castro Álava, Autores e impresores tudelanos. Siglos XV-XX (Pamplona 1963) 365-389; Id., Juan Antonio Fernández, un tudelano del siglo de la ilustración (Pamplona 1974); A. Canellas López, "Los estudios paleográficos en España y el archivero don Juan Antonio Fernández", Paleographica diplomatica et archivistica: studi in onore di Giulio Battelli (Roma 1979) 617-633; L. Moreno Garzón, "El manuscrito sigilográfico de D. Juan Antonio Fernández", Actas del Primer Coloquio de Sigilografia. Madrid, 2 al 4 de abril de 1987 (Madrid 1991) 353-359; M. a Á. Colomo de Granda, M. a P. Jiménez Zamora, "Juan Antonio Fernández y los manuscritos del autor conservados en la biblioteca pública 'Yanguas y Miranda' de
} 
temprana afición a la lectura de viejos documentos y de su familiaridad con los archivos eclesiásticos de su tierra, lo que orientaría de manera absoluta su trayectoria profesional. Casado con Francisca Uxaravi y Uxué -que le acompañó a todos los lugares en que fue requerida su experiencia archivística-, su vida cambiaría para siempre el día en que Antonio Jerónimo Tavira y Almazán (Iznatoraf, 1737-Salamanca, 1807) se convirtió en prior del monasterio de Uclés ${ }^{6}$.

Tavira no era un prior más de los muchos que se habían sucedido al frente del conventual santiaguista. Pasaba por ser uno de los mayores intelectuales de su tiempo, reformador, hombre ilustrado, miembro de número de la Real Academia Española desde 1775, amigo de personajes como Melchor Gaspar de Jovellanos, capellán de honor y predicador del rey Carlos III desde 1777 y asiduo de las más renombradas tertulias intelectuales del Madrid de su tiempo, especialmente de la que reunía en su casa María Francisca de Sales Portocarrero, Condesa de Montijo ${ }^{7}$. El priorato de Uclés significaba un paso importante en la carrera del intelectual jiennense, que veía así recompensada su cercanía a los círculos reformadores de la Corte mediante un destino que no le alejaba

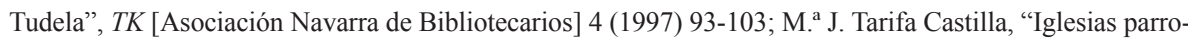
quiales de Tudela desaparecidas", Príncipe de Viana 66.234 (2005) 15-52; J. M. Abascal, R. Cebrián, Manuscritos sobre antigüedades de la Real Academia de la Historia (Madrid 2005) 204-209; F. Mikelarena Peña, "Los manuscritos de la biblioteca de Juan Antonio Fernández, erudito tudelano", Príncipe de Viana 67.237 (2006) 273-299; J. A. Yevés Andrés, “Estudio y edición de las observaciones sobre la elaboración, conservación, variantes y ortografía de los códices medievales, escritas por Juan Antonio Fernández Pascual en la última década del siglo XVIII", VI Jornadas Cientificas sobre Documentación Borbónica en España y América (1700-1868) (Madrid 2007) 433-470; F. Mikelarena Peña, "Los libros de historia de la biblioteca de Juan Antonio Fernández, erudito tudelano y académico correspondiente de la RAH", Príncipe de Viana 69.244 (2008) 459-495; Id., "Los libros de historia de la biblioteca de la sociedad tudelana de deseosos del bien público", Cuadernos de Investigación Histórica 25 (2008) 363-414; J. M. Abascal, R. Cebrián, Los viajes de José Cornide por España y Portugal de 1754 a 1801 (Madrid 2009) 55, 62-66, 181, 792, 818, 841; J. M. Abascal, "Los estudios epigráficos en Hispania (1756-1920). Un apunte desde los fondos manuscritos de la Real Academia de la Historia", Hispaniae. Las provincias hispanas en el mundo romano (Tarragona 2009) 108-111 y 113; F. Mikelarena Peña, "Los manuscritos y los libros sobre la historia de Aragón y Navarra de la biblioteca de Juan Antonio Fernández, erudito tudelano", Vasconia. Cuadernos de historia-geografia 37 (2011) 151-167.

${ }^{6}$ Tras este priorato de Uclés sería sucesivamente Obispo de Canarias (1791), obispo electo de Osma (1796) y Obispo de Salamanca (1798-1807). Falleció el 7 de enero de 1807 y fue enterrado en la capilla mayor de la catedral de Salamanca. Cf. la nota 8.

${ }^{7}$ Sobre su figura, cf. especialmente P. de Demerson, María Francisca de Sales Portocarrero, Condesa de Montijo. Una figura de la ilustración (Madrid 1975). Por su tertulia desfilaron gentes como Jovellanos, Francisco de Cabarrús, Pedro de Silva (hermano del Marqués de Santa Cruz), Juan Meléndez Valdés, Llaguno y Amírola, Martín Fernández Navarrete, Juan Pablo Forner, los Iriarte, el arquitecto Pedro Arnal, Mariano Luis de Urquijo o José Mazarredo entre muchos otros, incluyendo a pintores como Goya y Bayeu. Sobre esa tertulia, cf. J. Vargas Ponce, Disertación sobre las corridas de toros compuesta en 1807 por el capitán de fragata D... Edición ordenada y revisada por Don Julio F. Guillén y Tato (Madrid 1961) XVI-XVII; P. Demerson, op. cit. 101-123; V. Palacio Atard, "Cultura y sociedad en el setecientos madrileño", A. López Gómez (coord.), Madrid desde la Academia (Madrid 2001) 261-262; P. Pérez Cantó, E. Mó Romero, “Las mujeres en los espacios ilustrados”, Signos históricos 13 (2005) 52-55; J. Álvarez Barrientos, Los hombres de letras en la España del siglo XVIII: apóstoles y arribistas (Madrid 2006) 114. 
excesivamente de Madrid y le ponía al frente de la matriz santiaguista, en cuya orden militaba desde $1761^{8}$.

La llegada de Tavira supuso un revulsivo para la historia del viejo monasterio. $\mathrm{Su}$ archivo, que albergaba miles de documentos y cuyo contenido era casi desconocido por ser muy antiguos sus índices y no haberse realizado nunca un inventario exhaustivo que se pudiera considerar suficiente ${ }^{9}$, concentró el interés que quien ya entonces tenía una fantástica biblioteca personal y de quien confería a los documentos una importancia que habían obviado sus predecesores.

Eso explica que una de las primeras medidas de Antonio Tavira en Uclés fuera reclamar la presencia de Juan Antonio Fernández Pascual, el archivero tudelano de quien los obispos de su tierra ya conocían la experiencia en el manejo y archivo de documentos ${ }^{10}$, que debía enfrentarse a uno de los mayores retos profesionales de su carrera aún a costa de mudar su residencia durante unos años desde las vegas navarras a los umbrales de La Mancha.

Habiendo sido llamado su referido marido a efecto de arreglar el archivo de Santiago en Uclés, lo hizo así, haviéndolo acompañado la declarante y permanecieron por espacio de quatro años ${ }^{11}$.

\footnotetext{
${ }^{8}$ La figura de Tavira ha sido objeto de numerosos trabajos, entre los que cabe destacar los de M. Martes, Elogio fúnebre del... señor Antonio Tavira y Almazán, del Consejo de S.M., obispo de Salamanca. 7 de septiembre de 1807 (Salamanca 1807); B. Hernández, "La correspondencia de Pérez Bayer, Risco y Cornide con Antonio Tavira”, Boletín del Centro de Estudios del Siglo XVIII 10-11 (1983); J. Saugnieux, La Ilustración cristiana en España. Escritos de D. Antonio Tavira, obispo de Salamanca (1737-1807) (Salamanca-Oviedo 1986), e id., Un Prélat éclairé: Don Antonio Tavira y Almazán (17371807). Contribution à l'étude du jansénisme espagnol (Toulouse 1970); J. A. Infantes, Crisis religiosa e Ilustración, un horizonte desde la biblioteca de Tavira (Las Palmas de Gran Canaria 1981); Id., Tavira, ¿una alternativa de Iglesia? (Córdoba 1989); J. A. Infantes Florido, Diario de Tavira (Córdoba 1998).

${ }^{9}$ De esos intentos anteriores dio noticia Lorenzo Hervás y Panduro, Descripción del Archivo de la Corona de Aragón existente en la ciudad de Barcelona y noticia del Archivo General de la Militar Orden de Santiago existente en su Convento de Uclés (Cartagena 1801) 47-48: En un índice del archivo se lee lo siguiente: "Año 1505: índice del archivo general formado por virtud de provisión del Rey Don Fernando el católico". Últimamente en el año 1721 el augusto Rey Don Felipe V nombró a Don Luis de Salazar y Castro para arreglar el dicho archivo; pero Salazar falto de tiempo, o quizá llevado de su inclinación a las historias genealógicas, solamente arregló el archivo llamado de las pruebas de los caballeros. Sobre ese trabajo de Hervás, cf. el manuscrito RAH-9/5933/6 de José Cornide: Informe de Don José Cornide sobre la carta del abate Hervás impresa y no publicada en la que da cuenta de los papeles contenidos en los archivos de Barcelona y Uclés (Madrid 11 de diciembre de 1801); de la existencia de este manuscrito ya se hizo eco Fort y Pazos (C. R. Fort y Pazos, Discurso en elogio de Don José Cornide de Saavedra. Secretario que fue de la Real Academia de la Historia, leido en la Junta Pública que celebró este Cuerpo á 7 de junio de 1868, por su individuo de número y bibliotecario Don ---- [Madrid 1868] 50, n. ${ }^{\circ}$ 33).

${ }^{10}$ Así lo decía el propio Tavira: ... Don Juan Antonio Fernández, archivero del Reverendo Obispo de Tudela que, cediendo a mis repetidas instancias, dejó su casa y las comisiones en que estaba empleado... (A. Tavira, RAH-9-5933-7a).

${ }^{11}$ M. Sáinz, Apuntes... (op. cit. en nota 5) 398. Transcrito también en F. Mikelarena Peña, "Los manuscritos de la biblioteca...”, (op. cit. en nota 5) 275. Los datos proceden del inventario de bienes del fallecido, que se conserva en el Archivo de Protocolos Notariales de la Merindad de Tudela, Notaría de Tudela, escribano Francisco Urquiza, 1814, documento 161.
} 
Así lo contaría Francisca Uxaravi, la esposa de Juan Antonio Fernández, que muchos años después relataría con detalle las etapas del viaje desde Tudela ${ }^{12}-$ de donde partieron el 1 de agosto de 1789- hasta su llegada a primera hora de la mañana de aquel caluroso 8 de agosto al Sacro, Real y Militar convento de Uclés ${ }^{13}$.

Una de las primeras noticias sobre la presencia en Uclés del tudelano se conserva en la Real Academia de la Historia en los diarios de viaje del coruñés José Cornide ${ }^{14}$, que estuvo en el monasterio poco después de comenzar los trabajos de ordenación de su archivo:

En esta villa hallé a D. Juan Antonio Fernández, natural de Tudela, que está componiendo el archivo general de la Orden por encargo del Consejo de Órdenes; es muy inteligente en papeles y antigüedades; me obsequió y pasamos juntos a Cabeza del Griego, que dista legua y media ${ }^{15}$.

El trabajo de Fernández en Uclés no pasó desapercibido en los círculos intelectuales de la Corte. La importancia de los manuscritos del archivo lo justificaba, máxime si se tiene en cuenta que Ambrosio de Morales había descrito someramente algunas piezas originales del siglo XII de las que Fernández podía corroborar la existencia, tal y como hizo en las anotaciones al manuscrito escurialense que le envió Benito Cano para publicar ${ }^{16}$. Muy pronto, Fernández comenzó a cartearse con historiadores, cronistas e incluso políticos de su tiempo, a los que la información contenida en Uclés interesaba por diversos motivos ${ }^{17}$; el tudelano fue

12 J. R. Castro Álava, Autores... (op. cit. en nota 5) 110-111.

${ }^{13}$ J. A. Fernández, Noticia de la escabacion hecha en el territorio que llaman Cabeza del Griego y sus descubrimientos, copiados y explicados por..., año de 1790. Ms. RAH-9-5597, p. 3, que confirma la fecha.

${ }^{14}$ Cornide fue asiduo del conventual de Uclés, que convirtió en parada obligada en algunos de sus viajes. Allí había conocido el archivo de la mano de Juan Antonio Fernández entre 1789 y 1790 y volvió reiteradamente -años después y ya ausente el navarro- a descansar y revisar documentos. Las estancias posteriores documentadas son las de la primavera de 1794 (cf. J. M. Abascal, R. Cebrián, Los viajes... [op. cit. en nota 5] 67), agosto de 1797 (Ibid., 71 y 249), octubre y noviembre de 1801 (Ibid., 132-133, 825, 838 y 839: En Uclés he pasado el tiempo ya en la librería del convento, ya repasando manuscritos del Sr. Agurleta, que trató de las antigüedades y privilegios de la Orden, y otros del archivo entre los cuales he visto una provisión de el Sr. Felipe 2. ', librada en el año de 1567, por la cual nombra a Gaspar de Vega su Maestro mayor y trazador para que haga las plantas de la iglesia y convento, por estar ya vieja y ruinosa).

${ }^{15}$ Cornide, ms. RAH-9/3895/9; J. M. Abascal, R. Cebrián, Los viajes... (op. cit. en nota 5) 181.

${ }^{16}$ A. de Morales, "Noticias históricas..." (op. cit. en nota 2) 7-12.

${ }^{17}$ Valga como ejemplo el legajo de correspondencia conservado en la Biblioteca Nacional (B.N.E. Mss./17483, procedente de la colección de Pascual de Gayangos): Correspondencia de Juan Antonio Fernández, archivero general de la Orden de Santiago [fechadas entre 1777 y 1814]. Estas 177 hojas contienen cartas de Manuel Abella, Pedro Cevallos Guerra, José Cornide, Isidro Dolz, Francisco Hurtado de Mendoza, Miguel Iturbide, Juan Antonio Llorente, José María Magallón y Mencos (Marqués de San Adrián), José Moñino Redondo (Conde de Floridablanca), Lorenzo Prestamero, Manuel Risco, el propio Antonio Tavira, Joaquín Traggia de Santo Domingo y José Vargas Ponce. Dos de esas cartas - de Risco y Llorente respectivamente- están transcritas en una nota anónima titulada "Variedades. Dos cartas inéditas, dirigidas a D. Juan Antonio Fernández, archivero de Uclés", Boletín de la Real Academia de la Historia 14 (1889) 105-107. 
admitido de facto en ese selecto grupo de historiadores que discutían vigorosamente los textos de Plinio el naturalista, que especulaban sobre los trayectos de las vías romanas y que, por supuesto, tenían en la historia del primitivo cristianismo peninsular una fuente inagotable de conversación, fundamentalmente porque todos eran deudores en mayor o menor medida de La España Sagrada de Enrique Flórez que, como ellos, había tenido en la Real Academia de la Historia el referente institucional de sus quehaceres.

En 1793, Juan Antonio Fernández hacía sabedor a Benito Cano del desorden secular del archivo que Ambrosio de Morales se había encontrado dos siglos antes ${ }^{18}$. Había llegado el momento de enmendar aquella situación y de responder adecuadamente a las expectativas de Antonio Tavira, que había conseguido el nombramiento de Fernández por parte del Consejo de Órdenes. Para ello, además del auxilio que pudieran prestarle otros residentes en el monasterio, contó durante 13 meses con la ayuda de su cuñado Manuel Uxaravi ${ }^{19}$.

Sólo dos meses después de la llegada de Juan Antonio Fernández a Uclés y como consecuencia de haberse comenzado a registrar su copioso Archivo ${ }^{20}$, entre otras preciosas memorias se hallaron algunas relativas a una grande Población que hubo en el territorio que llaman Cabeza del Griego, no lexos de este Convento ${ }^{21}$, en el lugar en que había aparecido la inscripción o epitafio de un Sefronio Obispo descubierta en el mismo territorio el año $1760^{22}$.

Tales noticias interesaban por igual a Tavira y a Fernández. Para el primero, cualquier evidencia epigráfica o arqueológica que sirviera para completar la documentación episcopal y conciliar editada por Flórez habría de granjearle nuevas simpatías en los círculos madrileños, al tiempo que contribuiría a reforzar la confianza de quienes le habían puesto al frente del convento de Uclés. Para Fernández, era la oportunidad de sacar partido de sus trabajos en el archivo y de demostrar la eficacia de su labor. La historia del episcopado visigodo era además, para ambos, un terreno ignoto y ajeno a sus ocupaciones diarias en el que no debió faltar un componente lúdico que explica su actuación posterior.

El conocimiento, y buen gusto que el dicho Prior tiene de la venerable antigüedad, y de todo género de literatura, no le dexaron libertad para mirar con indiferencia una especie, que averiguada podía servir de

\footnotetext{
${ }^{18} \mathrm{Cf}$. nota 3.

${ }^{19}$ J. R. Castro Álava, Autores... (op. cit. en nota 5) 112.

${ }^{20}$ J. A. Fernández, Noticia de la escabacion... (op. cit. en nota 13) 3.

${ }^{21}$ Ibid., 3-4.

${ }^{22}$ Ibid., 5 y 22. La fecha está confirmada en J. Capistrano de Moya., Apéndice i demostración del día y año de la muerte del Sefronio obispo Santo de Segobriga (Alcalá de Henares 1795) 4; M. López, Memorias históricas de Cuenca y su obispado (Madrid 1949/53) vol. I (edición de A. González Palencia a partir del manuscrito Memorias y relaciones históricas, topográficas, civiles y eclesiásticas de la ciudad de Cuenca, de su obispado y provincia, Cuenca ms. circ. 1787) 287-288.
} 
ilustración a la Historia civil y Eclesiástica de estos Reynos. A esto se aumentaba ser el terreno donde decían estubo fundada la mencionada Población, propio de la misma Orden y de su Mesa Magistral. La proximidad que tiene a este convento era también motivo para que el Prior pasase a reconocer el sitio. En fin, resolvió verlo y registrarlo, como se verificó en el día diez y siete de Octubre del año referido [1789]. La multitud de ruinas y vestigios que hay en aquel sitio dan bien a entender que allí estubo alguna populosa ciudad, y así dio orden para que se hiciese Escabación ${ }^{23}$.

Lo que Fernández relataría en ese documento era el inicio de las excavaciones en la basílica hispano-visigoda de Segobriga, que habrían de servir para descubrir un considerable número de epitafios episcopales visigodos y una cantidad aún superior de inscripciones de época romana. Unos y otros cambiarían los conocimientos futuros sobre la historia de la región.

A propósito de aquel primer viaje, diría Fernández:

Sabiendo el Alcalde y Cura de Sahelices que el dicho Prior de Uclés estaba haciendo este reconocimiento en aquel vecino territorio, vinieron a presentarle una piedra de alabastro quebrada con las primeras líneas de una inscripción gótica que daba noticia de un Obispo virtuoso llamado Sefronio, la qual en el año de 1760 fue descubierta casualmente en medio de las llanuras expresadas ${ }^{24}$.

Los personajes identificados como "Alcalde y Cura de Sahelices" eran Vicente Martínez Falero, cuya familia asumiría como propia la protección de las excavaciones hasta el punto de llegar a escribir algunas páginas sobre los hallazgos en las Memorias de la Real Academia de la Historia ${ }^{25}$, y Bernardo Manuel Cossío, que disputaría por escrito sobre la santidad de los personajes mencionados en los epitafios visigodos ${ }^{26}$.

Es decir, un prior santiaguista, un archivero tudelano, el alcalde de una pequeña localidad manchega y su párroco acabaron convirtiéndose en arqueólogos e intérpretes de las evidencias obtenidas en las excavaciones, definitivamente conocidas en Madrid tras saberse que el 14 de diciembre de 1789 se habían descubierto -en presencia de Tavira y Fernández- varios osarios identificados por

${ }^{23}$ J. A. Fernández, Noticia de la escabacion... (op. cit. en nota 13) 4-5.

${ }^{24}$ J. A. Fernández, Noticia de la escabacion... (op. cit. en nota 13) 21-22.

${ }^{25}$ J. F. Martínez Falero, "Impugnación al papel que con título de Munda y Cértima celtibéricas dio a luz el R. P. M. Fr. Manuel Risco, del Orden de S. Agustín remitida en 27 de junio de 1802 a la Real Academia de la Historia por su individuo correspondiente D. Juan Francisco Martínez Falero, abogado de los Reales Consejos, vecino de la villa de Saelices", Memorias de la Real Academia de la Historia 4 (1805) 1-73.

${ }^{26}$ Ms. RAH-9/5939/12, con las observaciones remitidas por Cossío sobre los descubrimientos de antigüedades en Cabeza del Griego. 
laudas sepulcrales visigodas en las que se mencionaba a diversos obispos de la sede segobrigense $\mathrm{e}^{27}$. El propio Fernández redactó aquella misma tarde en su celda de Uclés un acta notarial con el relato de los acontecimientos ${ }^{28}$, documento que luego firmarían todos los testigos, y asumió directamente el mayor protagonismo en los trabajos arqueológicos, como más tarde nos haría saber:

Yo me he hallado también presente a ellos [se refiere a los descubrimientos], y he pasado varias veces al expresado territorio, y he copiado, ya que no con primorosa delicadeza, con la mayor fidelidad y atención escrupulosa, las inscripciones y monumentos que, acompañados de una circunstanciada relación, fueron remitidos a S. M. por mano del Excmo. Sr. Conde de Floridablanca, a quien los dirigió el referido Prior, y de Orden Real pasaron los diseños y sus explicaciones a la Real Academia de la Historia $^{29}$.

Por si tras leer el párrafo anterior albergáramos aún alguna duda sobre ese protagonismo arqueológico y epigráfico del tudelano, éste añadió en una carta de 1792 que Todo lo demás remitido por la Secretaría de Estado a la Real Academia de la Historia es obra mía ${ }^{30}$. Es decir, toda la documentación que conservamos prueba sin lugar a dudas que fue Juan Antonio Fernández el autor físico de las excavaciones en la basílica visigoda de Cabeza del Griego entre finales de 1789 y comienzos de 1790, pese a que el tiempo y las envidias personales le relegaran al olvido, hasta el punto de que su nombre no apareció en las dos inscripciones -latina y castellana- que se pusieron en el lugar para conmemorar las excavaciones y para identificar el muro que había de proteger las ruinas ${ }^{31}$; ni siquiera las noticias

${ }^{27}$ Omitimos el relato detallado del viaje a Segobriga y de las primeras excavaciones, financiadas en parte por Antonio Tavira, asuntos que hemos comentado ampliamente en J. M. Abascal, "Los estudios epigráficos en Hispania (1756-1920). Un apunte desde los fondos manuscritos de la Real Academia de la Historia", Hispaniae. Las provincias hispanas en el mundo romano (Tarragona 2009) 108-110; cf. también J. M. Abascal, R. Cebrián, Los viajes... (op. cit. en nota 5) 62-64.

${ }^{28} \mathrm{RAH}-11 / 8167 / 74$ con el acta del hallazgo de las reliquias (autógrafo firmado; un pliego en fol. con 4 páginas sin numerar. Hay copias en RAH-CU/9/7953/1-11 y RAH-CU/9/7953/1-12). Junto a ese Acta se conservan algunos documentos que guardan relación con el mismo suceso: RAH-11/8167/75 (Huesos que quedan depositados en el Caxoncito correspond(ien)te a Nigrino Obispo-De Sefronio Obispo; un fol. sin numerar); RAH-11/8109/4a (Acta de la invención de las Reliquias de Nigrino y Sefronio Obispo, en la Escabacion de Cabeza del griego en 14. de Dic(iembr)e de 1789; borrador original del documento RAH-11/8167/74).

${ }^{29}$ J. A. Fernández, Noticia de la escabacion... (op. cit. en nota 13) 6.

${ }^{30}$ Carta de Fernández a Manuel Losada y Quiroga. Uclés, 19 de septiembre de 1792.

${ }^{31}$ Texto latino: Anno reparatae salutis MDCCLXXXX Antonius Tavira prior Uclensis Episcopus canariensis designatus Emmanuel Bernardus de Cossio Sahelicensis parochus Joannes Franciscus Falero et Vicentius Martinez Falero eiusdem oppidi nobiles cives vetustissimum hoc Segobrigensium cementerium barbarorum strage collapsum et congestis ruderibus prorsus obrutum propriis impensiis effodiendum curarunt Carolus IIII p. p. memoriae apud posteros diuturnioris ergo et ne rursus praestantissimum monumentum iniuria temporum aboleret publicis sumptibus muro cingi ac servari iussit (RAH-9/5597/2). Texto castellano: Reynando l. c. R. M. del S(eñ)or D(o)n Carlos IIII y siendo Alcaldes 
posteriores de las excavaciones le harían justa memoria, como sabemos por relatos relativamente próximos a sus trabajos en Segobriga ${ }^{32}$. En vista de esta situación, como único testigo directo de los descubrimientos, Juan Antonio Fernández optó finalmente por escribir el 4 de febrero de 1791 a Felipe Solano, obispo de Cuenca, en estos términos:

El haber intervenido yo en la excavación hecha en el territorio llamado Cabeza del Griego ... puede ser motivo para que me supongan autor de algunos yerros que no he cometido, y que los inteligentes notarán en ciertos papeles que se van esparciendo y andan ya en manos de muchos, donde hay vertidas noticias y especies mías de que se han aprovechado, callando mi nombre; pero ésta que parece ingratitud, me es por otra parte favorable, por cuanto no me atribuyen los defectos que voy a anotar y son dignos de corrección... ${ }^{33}$.

La documentación de aquellos trabajos que ha llegado hasta nosotros incluye un auténtico diario de excavaciones con multitud de apuntes, bocetos, indicaciones de hallazgos, etc. ${ }^{34}$, que va acompañada de diversas notas sobre los hallazgos $\mathrm{y}$ dibujos de inscripciones, hasta formar en su conjunto un primer ensayo arqueológico de lo que el propio Fernández identificaría como las ruinas de Segobriga y, al mismo tiempo, el primer esbozo de un catálogo de sus inscripciones.

De este modo, obligado por la necesidad de interpretar los numerosos textos latinos que se fueron descubriendo en las excavaciones, Fernández pasó en pocos meses de ser uno de los archiveros de mayor reputación de su tiempo a convertirse en epigrafista accidental.

de la villa de Saelices los s(eñor)es D(o)n Juan Fran(cis)co M(artí)n(e)z Falero y Diego de Platas Oliva se hizo esta cerca a expensas de el público para resguardo de este antiguo cementerio, que habiendo estado oculto entre sus ruinas, por espacio de mil ciento setenta y cinco años, fue descubierto por disposición y a costa del m(uy) ill(ustr)e s(eño)r D(o)n Antonio Tavira, prior de el R(ea)l Convento de Santiago de Uclés, Obispo electo de Canarias, de el Consejo de S. M., D(o)n Bernardo Manuel de Cossío, cura de la parroquial de d(i)cha villa, D(o)n Juan Francisco y D(o)n Vicente Martínez Falero (RAH-9/5597/3).

${ }^{32}$ Sólo nueve años después de concluidas las excavaciones de Tavira y Fernández, el nombre del tudelano había desaparecido, como demuestra la crónica de Lorenzo Hervás y Panduro, que estuvo en Uclés y Segobriga en el verano de 1799: Monseñor Tamayo desé que yo observase las ruinas de Segobriga nuevamente descubiertas por influjo y expensas de monseñor Tavira, del abogado Don Juan Falero, y de otros curiosos de Saelices; y con la dirección del dicho señor Falero, instruidísimo en todas las antigüedades descubiertas, fui a ver las ruinas en compañia de mi favorecedor el estudioso joven señor Don Tomas Bernad y Barreda (L. Hervás y Panduro, Descripción del Archivo... op. cit. en nota 9,68$)$.

${ }^{33}$ RAH-9/5597/2. El documento incluye la segunda Memoria de las excavaciones en Segobriga, redactada por Juan Antonio Fernández y firmada el 4 de febrero de 1791.

${ }^{34}$ J. A. Fernández ms.1789-1790, RAH-11/8109/4k. Se conserva también una Memoria final de los trabajos redactada en 1790 (RAH-11/8109/4b). 
Los apuntes de Juan Antonio Fernández y los informes que Tavira fue enviando al Conde de Floridablanca ${ }^{35}$-con dibujos e interpretaciones epigráficas salidas de manos del tudelano- hablan por sí solos de la eficacia y habilidad de éste a la hora de copiar las inscripciones que fue encontrando en las excavaciones y las que, ya por entonces, se encontraban empotradas en diversas casas de la cercana localidad de Saelices (fig. 2).

En poco más de cuatro meses, es decir, entre octubre de 1789 y febrero de 1790, por las manos de Fernández pasaron algo más de 60 inscripciones o fragmentos de éstas, que contribuirían a dar consistencia al capítulo dedicado por Hübner a Cabeza del Griego en la edición del Corpus inscriptionum Latinarum de 1869, a pesar de lo cual, por falta de evidencias, éste último se resistió a ubicar allí el solar de Segobriga y prefirió mantener Cabeza del Griego en la categoría de los oppida ignota.

Las notas de Fernández sirven para conocer el proceso de transcripción de los epígrafes, desde su recogida en la excavación de Cabeza del Griego o su copia en la localidad de Saelices, hasta su inclusión en los informes que Tavira enviaría al Conde de Floridablanca y que éste haría llegar a la Real Academia de la Historia.

Gracias a ese acopio de manuscritos que conservamos, estamos muy bien informados de ese proceso, que se sustanció fundamentalmente en tres series epigráficas que se realizaron en el mismo orden cronológico en que las presentamos:

1. Anotaciones de campo. Se trata de borradores de muy mala calidad incorporados tanto a las páginas de lo que consideramos propiamente un Diario de excavación ${ }^{36}$, como de apuntes de epígrafes sobre los que Fernández hizo varios bocetos con leves diferencias entre $\mathrm{si}^{37}$.

2. Dibujos de inscripciones realizados con mayor cuidado para ser incorporados a los informes que Antonio Tavira envió regularmente al Conde de Floridablanca entre finales de 1789 y comienzos de $1790^{38}$. Esta serie, de mucha calidad (fig. 2), constituye el conjunto que sería luego copiado y "duplicado"

${ }^{35}$ La relación de los informes puede verse en J. M. Abascal, R. Cebrián, Los viajes... (op. cit. en nota 5) 445-446.

${ }^{36}$ J. A. Fernández, ms. 1789-1790 RAH-11/8109/4k.

${ }^{37}$ J. A. Fernández, ms. 1789-1790 RAH-11/8109/4g o 11/8109/4i, por ejemplo.

${ }^{38}$ La referencia de los dibujos en el archivo de la Real Academia de la Historia es RAH-9/7953/1(31 a 50). Las fechas y referencias de esos informes, cuyos originales se guardan en la Academia, son las siguientes: 14 de diciembre de 1789 (RAH-9/7953/1-10), 30 de enero de 1790 (RAH-9/7953/1-25) y 3 de febrero de 1790 (RAH-9/7953/1-26). Una minuta de oficio de 23 de marzo de 1791 (RAH-9/7953/1-54) indica que se devolvieron al Conde de Floridablanca "los planos y diseños de las antigüedades recién descubiertas en el sitio llamado Cabeza del Griego, que de Orden de S. M. se sirvio V. E. remitir a la Academia Real de la Historia con fecha de 28 de enero, 4 y 17 de febrero del año próximo pasado" pero o bien esa devolución no llegó a producirse o bien fue incompleta, pues gran parte de esos "diseños de las antigüedades" salidos de la mano de Fernández siguen en el archivo de la Academia. A esta serie pertenecen las ilustraciones que conserva el Archivo Histórico Nacional (AHN Estado MPD 630-633 y 844). 


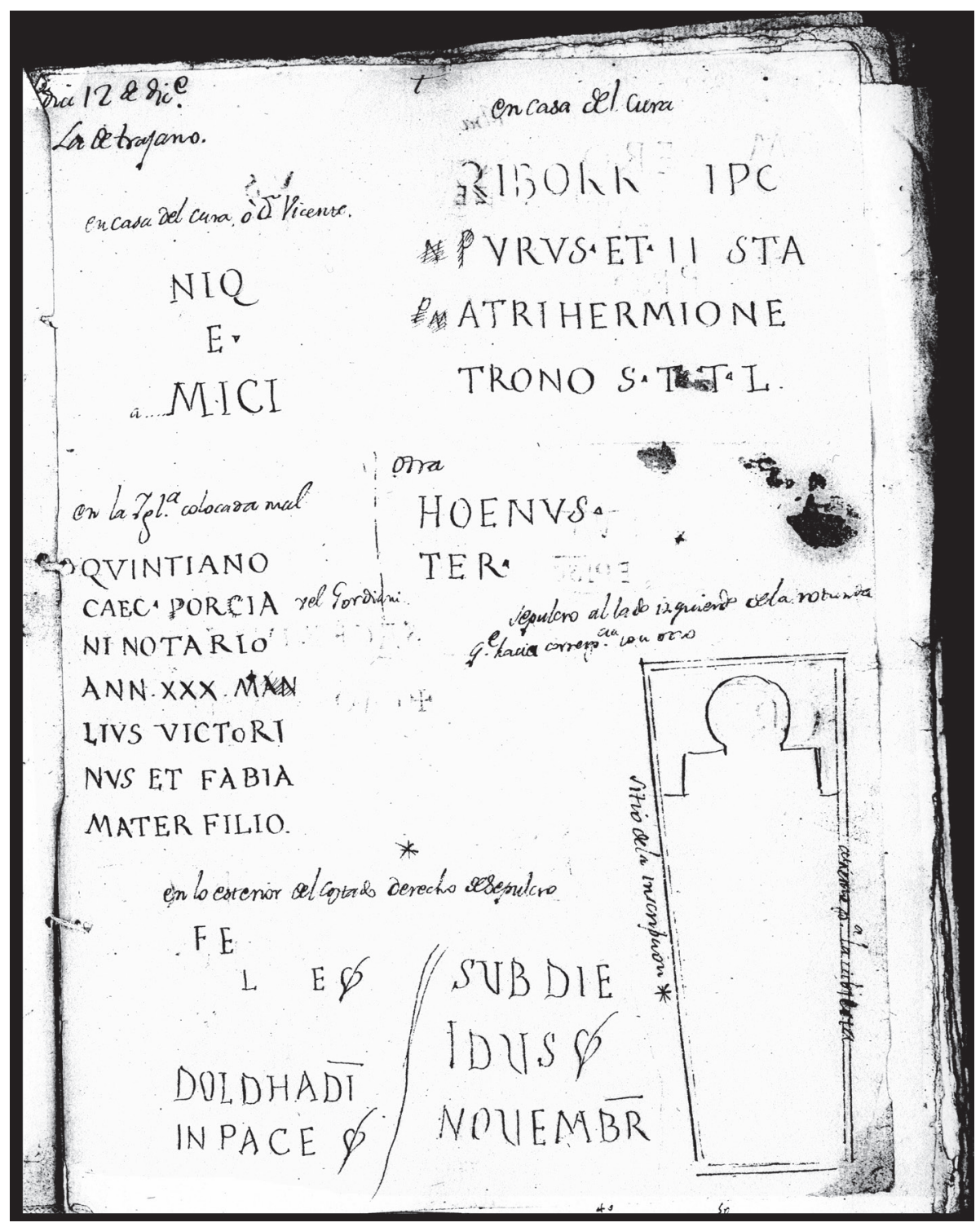

Figura 1. Juan Antonio Fernández, Imagen del documento que hemos considerado Diario de las excavaciones. RAH-11/8109/4k. En el ángulo superior izquierdo dice: Día 12 de dic(iembr)e, es decir, se trata de notas tomadas tanto en Saelices como en la propia excavación el 12 de diciembre de 1789. 
por Francisco Javier de Santiago Palomares ${ }^{39}$, dando así lugar a los documentos que forman la pieza RAH-9/5939/16 ${ }^{40}$, hoy encuadernada en un volumen facticio (fig. 4).

3. Dibujos incorporados por Juan Antonio Fernández al manuscrito en que daba cuenta de los trabajos arqueológicos realizados, y que lleva por título Noticia de la escabación hecha en el territorio que llaman Cabeza del Griego, y sus descubrimientos, copiados y explicados por D. Juan Antonio Fernández, \&c. Año de $1790^{41}$. Casi todas las ilustraciones están colocadas al final del manuscrito, en donde las inscripciones ocupan las páginas 147-155 (fig. 4).

Pese a disponer de un acervo epigráfico tan considerable, Juan Antonio Fernández no pasó de describir las evidencias que el destino había puesto a su alcance y de indicar los lugares en que se conservaban. Carecía de formación específica en epigrafía aunque, como buen latinista, podía dar sentido a los textos que reconoció. Tanto Tavira como él estaban interesados fundamentalmente en las laudas episcopales visigodas que se habían descubierto, pues con ellas era posible discutir la antigüedad de la sede de Segobriga y tratar de situar en el tiempo a estos prelados; es decir, les interesaba -como a quienes les acompañaron en las excavaciones- las novedades relacionadas con la historia de la Iglesia, lo que dejaba las antigüedades romanas en un segundo lugar, casi anecdótico, sin que ello supusiera silenciar esos descubrimientos; el rigor documental de Juan Antonio Fernández hizo que se tomara puntual nota de todos esos descubrimientos aunque en ninguno de los informes que se elaboraron en los meses siguientes se hizo la más mínima referencia al significado de estos epígrafes. Precisamente por eso, Juan Antonio Fernández no pasó de ser un epigrafista accidental.

Desconocemos la fecha exacta en que se decidió suspender los trabajos de excavación en la llamada basílica visigoda de Cabeza del Griego, el lugar en que se había desarrollado casi toda la actividad arqueológica de Tavira y Fernández.

${ }^{39}$ Francisco Javier de Santiago Palomares (Toledo, 5 de marzo de 1728-Madrid, 13 de enero de 1796) fue bibliotecario de la Real Academia de la Historia desde el 2 de diciembre de 1794, tesorero de la misma desde el 28 de noviembre de 1794 y Primer Oficial Archivero del Reino hasta su muerte. Hübner (CIL II, p. 112), le llama homo modestissimus, inter paleographos hispanos primarius, sed ignotus. Fue uno de los mejores dibujantes y calígrafos de que dispuso la Academia en la segunda mitad del siglo XVIII para duplicar aquellos documentos y dibujos que había que devolver y de los que se quería guardar copia, una práctica habitual de la época. Además de las muchas copias que hizo, fue autor de una Polygraphia gothico-española. Origen de los caracteres o letras de los godos en España; su progresso, decadencia, y corrupción desde el siglo V hasta fin del XI en que se abrogó el uso de ellos, y substituyó la letra gótico-francesa. La obra se conserva en forma de manuscrito, fechado en el año 1764, en el archivo

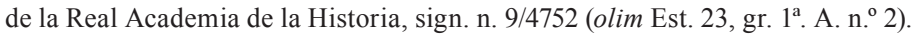

${ }^{40}$ Las entregas de dibujos originales a Francisco Javier de Santiago Palomares por parte del Secretario de la Academia, Antonio Capmany, se prolongaron hasta el 3 de marzo de 1790, como consta de la documentación conservada (RAH-9/7953/1-20).

${ }^{41}$ RAH-9/5597/1 (autógrafo; un cuaderno en 4. ${ }^{\text {o }} 160$ páginas numeradas 1-159 con 17 láminas); borrador incompleto en RAH-11/8109/4b. 


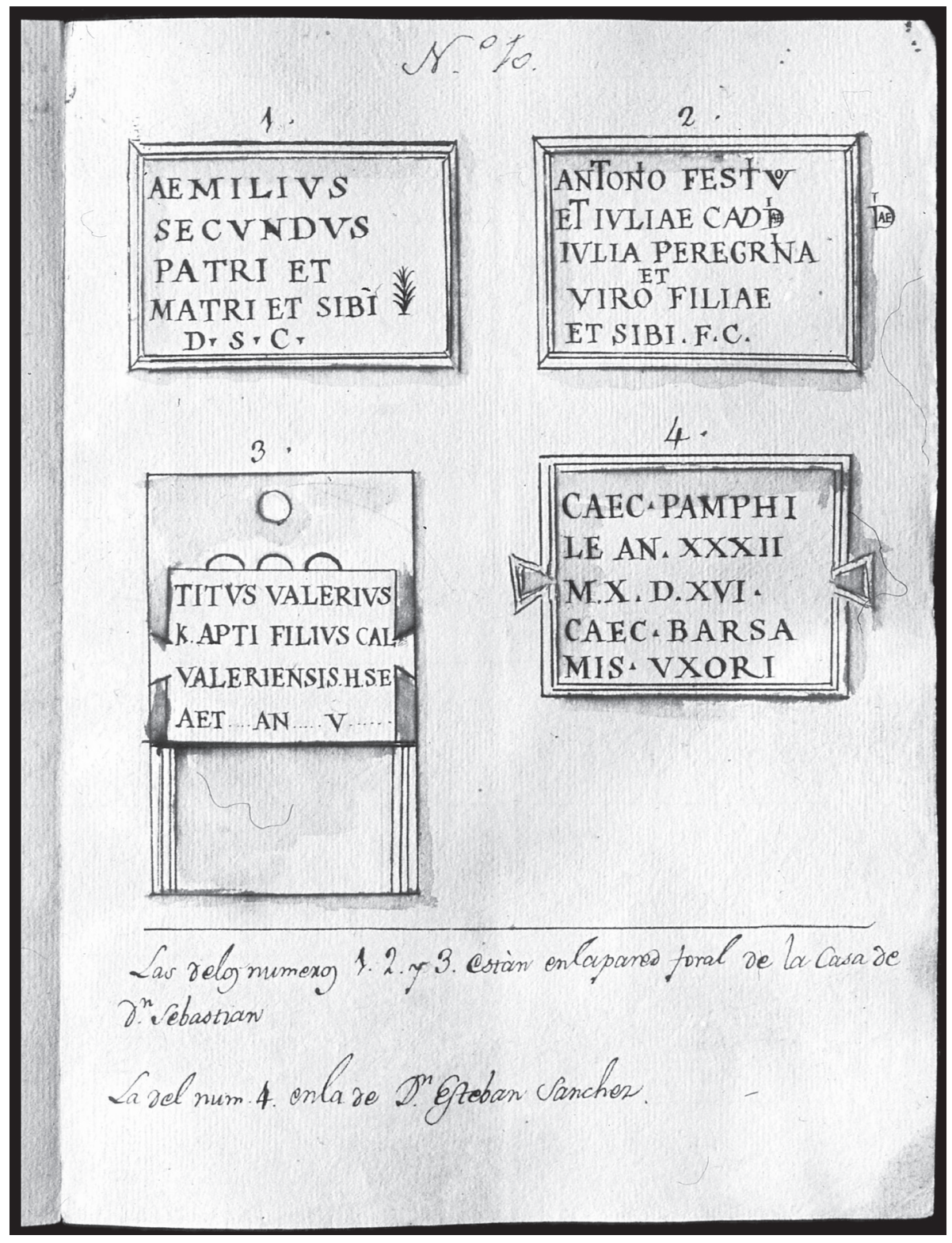

Figura 2. Juan Antonio Fernández, Inscripciones colocadas en varios edificios del lugar de Sahelices, copiadas en 13 de noviembre de 1789. RAH-9/7953/1(38), original sobre a partir del cual se obtuvo la copia de RAH-9/5939/16 (cf. Figura 3). Foto: J. M. Abascal. 
Sirvan como indicios el hecho de que el último de los informes conservados de los que Antonio Tavira envió al Conde de Floridablanca lleva fecha del 3 de febrero de $1790^{42}$ y que la Noticia de la escabación... escrita por Fernández (cf. supra) está escrita ese mismo año. Es decir, hay que suponer que los trabajos terminaron a finales del invierno de 1790 y que esa primavera Juan Antonio Fernández se reincorporaría plenamente a los trabajos de catalogación del archivo del conventual santiaguista, la labor que le había traído a tierras conquenses. No obstante, el análisis y la interpretación de los documentos por parte de los miembros de la Real Academia de la Historia se prolongaría aún durante un año, pues la minuta de oficio que habla de la devolución de los dibujos originales está fechada el 23 de marzo de $1791^{43}$. Nótese, además, que el informe final enviado por el propio Fernández a la Academia $^{44}$-identificado como Papel ó Coleccion de noticias de la Escabacion de las ruinas de la antigua Segobriga en el territorio de Cabeza del Griego- iba acompañado de un oficio ${ }^{45}$ fechado en Uclés el 22 de febrero de 1791. Aquel mismo año, Antonio Tavira sería nombrado Obispo de Canarias y debería ceder el testigo del priorato de Uclés; poco después de hacerlo "y estando para salir de la Península" a mediados del mes de noviembre ${ }^{46}$, recompensaría los servicios de Juan Antonio Fernández al firmar en Cádiz la recomendación oficial para que se nombrara Archivero General de la Orden de Santiago ${ }^{47}$.

Por lo que respecta a Fernández, fuera de algunas cartas ocasionales, poco sabemos de su labor durante los tres años que aún se prolongaría su estancia en Uclés. No obstante, las evidencias que conservamos de su trabajo en el archivo hablan por sí solas de ese eficaz trabajo que permitiría disponer de un inventario prolijo de aquel importante acerbo documental ${ }^{48}$. Nuestro mejor testigo de ello es Lorenzo Hervás y Panduro, que pasó allí dos semanas en 1799, pese a que -seguramente por desconocer su identidad-silenció el nombre del tudelano:

Mi actual pasajero domicilio en esta villa del Horcajo, sujeta en lo espiritual al Señor Obispo Prior de Uclés, me ha presentado la favorable

${ }^{42}$ RAH-9/7953/1-26. No consta que hubiera informes posteriores.

${ }^{43}$ RAH-9-7953/1-54.

${ }^{44}$ Se trata sin duda del informe ms. RAH-9/5597/1.

${ }^{45}$ RAH-11/8240/23a.

${ }^{46}$ RAH-9-5933-7a, página 1.

${ }^{47}$ Antonio Tavira, Copia del informe que el ilustrísimo señor Don Antonio Tavira y Almazán, Doctor Teólogo del claustro de la Universidad de Salamanca, Capellán de honor y Predicador de su Magestad, Prior que fue de la Real Casa de Santiago de Uclés, y hoy dignísimo Obispo de Canaria, dio al Real y Supremo Consejo de las Órdenes, recomendando el mérito contraído por Don Juan Antonio Fernández en el arreglo y coordinación del Archivo General de la Orden de Santiago. Cádiz, 19 de noviembre de 1791. Impreso, 2 páginas (RAH-9-5933-7a).

${ }^{48}$ El mismo Fernández dejaría redactado un informe que habría de servir de guía para el manejo de ese archivo: RAH-11-8167-70: Noticia del principio, progresos y ultimo estado del Archivo general de la Orden de Santiago; en el mismo legajo se conservan otras notas de Fernández que llevan por título Noticias sobre el Archivo de Uclés, Orden del Temple y otras con otros manuscritos. 


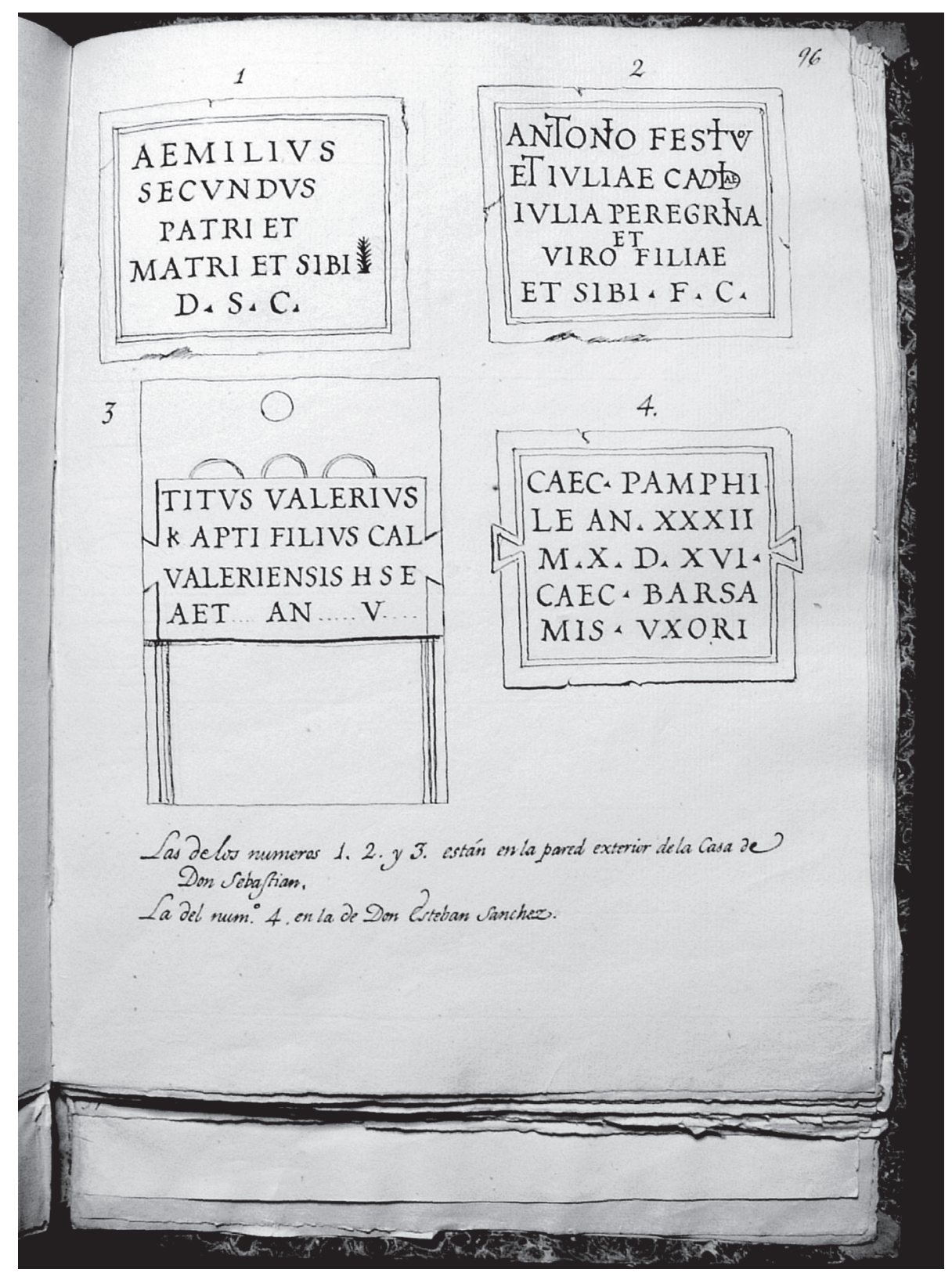

Figura 3. Francisco Javier de Santiago Palomares, Copias de los dibujos originales de las inscripciones romanas y antigüedades de Cabeza del Griego. RAH-9/5939/16, con copia de RAH-9/7953/1(38) (cf. Figura 2). Foto: J. M. Abascal. 
oportunidad de poder observar el excelente y bien ordenado archivo general que de la militar orden de Santiago hay en su convento magnífico de Uclés.... Éste se ha hecho con magnificencia, y se ha ordenado excelentemente por disposición de monseñor Don Antonio Tavira, actual Obispo de Salamanca, que siendo Prior del dicho convento de Uclés empleó millares de escudos en formar lo material del archivo, y en ordenar sus documentos haciendo copias de los que por su antigüedad o letra confusa eran casi ilegibles. Los volúmenes y escrituras están en cajoncitos de nogal con bronces dorados. Estos cajoncitos visten cuatro fachadas en una sala contigua a la iglesia de la parte del septentrión... No sin admiración y con gran placer observé la muchedumbre y buen estado de los volúmenes y escrituras que hay en el $\operatorname{archivo}^{49}$.

Precisamente ese silencio sobre la figura de Fernández sería el principal reproche que haría Cornide a este texto de Hervás cuando la Real Academia de la Historia le pidió que lo informara para publicarlo ${ }^{50}$.

Terminada su labor en Uclés, a mediados de mayo de 1793 Juan Antonio Fernández y su esposa debían abandonar el monasterio santiaguista para regresar a su tierra. Para entonces, Antonio Tavira ya estaba muy lejos de allí pero su influencia y su recomendación expresa de $1791^{51}$ fueron decisivas para que Fernández recibiera - dos años después de haberse solicitado- el título de Archivero general de la Orden de Santiago, firmado en Madrid por orden del rey Carlos III el 14 de mayo de $1793^{52}$.

Así fue como cuatro años después de su llegada a Uclés, Fernández y su esposa trasladaron su residencia a Zaragoza, donde el de Tudela debía ocuparse de ordenar el archivo de la Orden de San Juan que se guardaba en San Juan de los Panetes $^{53}$. No sabemos si entre Uclés y Zaragoza hubo una estancia -breve, muy breve $^{54}$ - en Tudela pero, en todo caso, la correspondencia de noviembre de 1793 ya está fechada en la capital aragonesa.

De esas cartas nos interesan especialmente las cruzadas con José Cornide en las que Fernández explicaba los progresos de sus estudios sobre Segobriga en los

${ }^{49}$ L. Hervás y Panduro, Descripción del Archivo... op. cit. en nota 9, 44-46.

${ }^{50} \mathrm{~J}$. Cornide, ms. RAH-9/5933/6: ... sólo hallo de menos que no advierta que el buen orden con que está arreglado en lo antiguo se debe al señor Agurleta, conventual de aquella ilustre casa y bien conocido por la publicación del Bulario de la orden, y en lo modeno a Don Juan Antonio Fernández, a quien empleó en este trabajo el señor Tavira.

${ }^{51}$ Cf. nota 47.

${ }^{52}$ Una copia impresa del título se conserva en la Real Academia de la Historia (RAH-9/5933/7b, f. 144); cf. también Castro 1941, 112-113.

${ }^{53}$ Según Francisca Uxaravi, su esposa, tras los cuatro años de Uclés residieron en Zaragoza "por espacio de diez y seis años arreglando el archivo de San Juan de los Panetes" (cf. nota 11).

${ }^{54}$ Nótese que algunos estudios biográficos les sitúan de nuevo residiendo en Tudela antes de pasar años después a Zaragoza. Ese intervalo es imposible, como sabemos ahora por los lugares en que se redactaron las cartas que conserva la Real Academia de la Historia. 


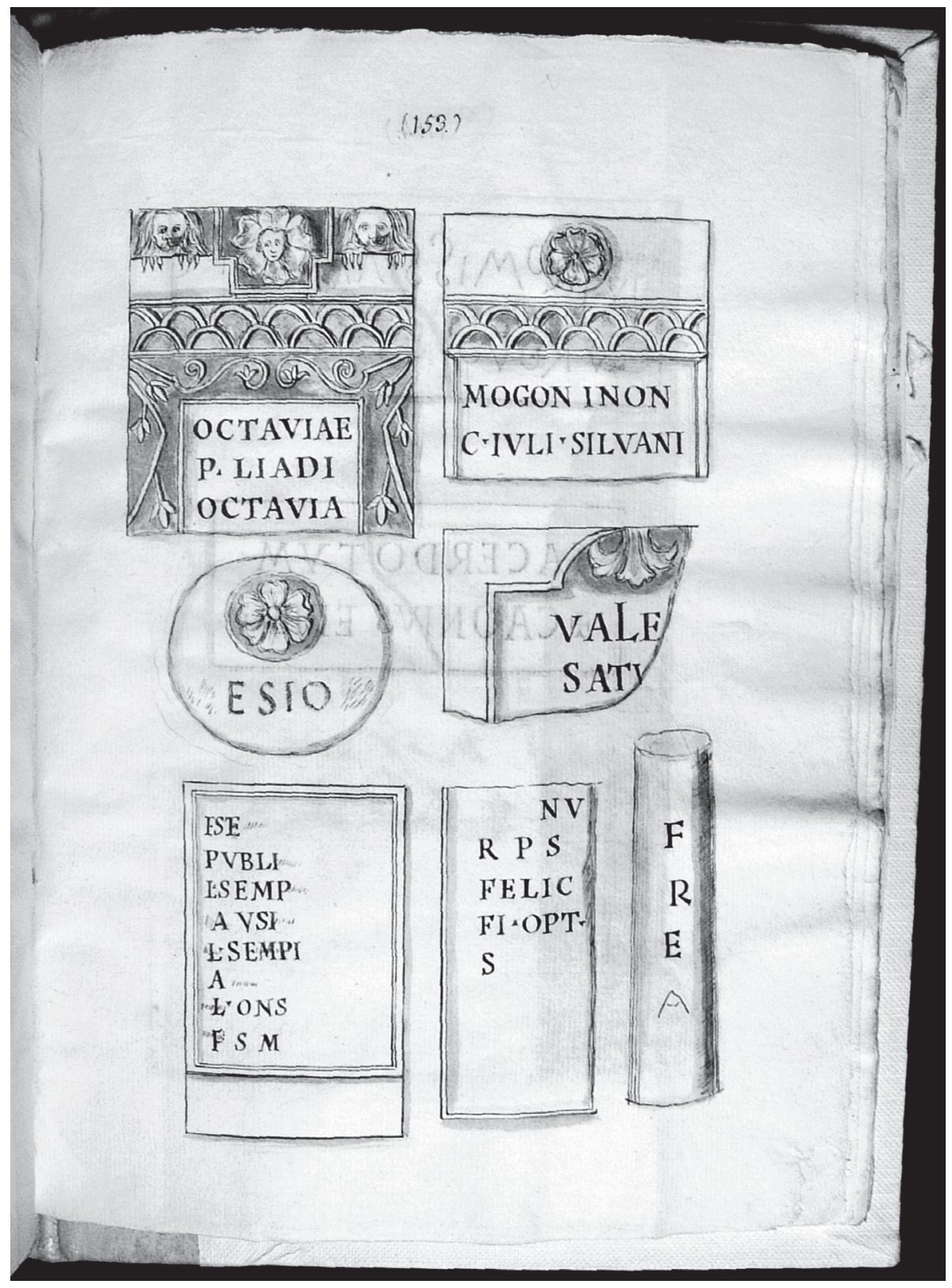

Figura 4. Juan Antonio Fernández, Noticia de la escabación hecha en el territorio que llaman Cabeza del Griego, y sus descubrimientos, copiados y explicados por D. Juan Antonio Fernández, \&c. Año de 1790. RAH sign. n. 9/5597/1, p. 153. Foto: J. M. Abascal. 
que continuaba interesado, pese a la lejanía temporal y geográfica. Las cartas y sus fechas consecutivas indican que seguía desarrollando una gran actividad sobre los hallazgos que había realizado en Segobriga: Zaragoza, 4 de noviembre de 1793 (... Aqui me están abriendo las laminas de la excavación de Cabeza del Griego, o Segobriga, con animo de dar a luz lo que tengo trabajado y recogido sobre este asunto... $)^{55}, 30$ de noviembre de 1793 (... es preciso decir algo más de lo que hasta aquí se ha escrito y enmendar a Masdeu, Traggia, etc. $)^{56}, 17$ de diciembre de $1793^{57}, 3$ de mayo de 1794 (He recogido en casa los papeles que tenia allá relativos a la excavación del terreno de Cabeza del Griego y los he traído conmigo para tenerlos presente y decir a Vd. en vista de ellos sobre este asunto; y me parece que se podrá decir bastante de nuevo $)^{58}$, etc. Las cartas de este tipo y con la misma temática se prolongarían aún entre la primavera de 1794 y el otoño de $1801^{59}$.

El traslado a Zaragoza había devuelto a Juan Antonio Fernández a su profesión de archivero, una actividad que había desarrollado meritoriamente en Uclés pero de la que había distraído tiempo en favor de la arqueología y la epigrafía. Ahora su tiempo discurría entre la capital aragonesa, donde continuaba con sus tareas en San Juan de los Panetes, y los viajes ocasionales a Tudela, donde la salud de su padre ocasionalmente reclamaba su presencia, tal y como explica en sus cartas.

Más allá de la continuidad de los estudios ocasionales sobre Segobriga, esporádicamente citados en la correspondencia, no tenemos noticias de que el de Tudela iniciara otros estudios arqueológicos antes de 1804. En agosto de ese año, aprovechando la ocasión de llevar poca agua el Ebro, se hicieron varios reparos en la deteriorada fábrica del magnifico puente de piedra y como consecuencia de ello se encontraron dos grandes tubos o caños de plomo que ya en otros años de sequía habían llegado a divisarse ${ }^{60}$. La intervención del intendente Blas Ramírez,

\footnotetext{
${ }^{55}$ RAH-9/3921/78.

${ }^{56}$ RAH-9/3921/79.

${ }^{57}$ RAH-9/3921/80.

${ }^{58}$ RAH-9/3921/81.
}

${ }^{59}$ Las cartas conservadas son las siguientes: 20 de mayo de 1794 (RAH-9/3921/83), 30 de septiembre de 1794 (RAH-9/3921/84), 2 de diciembre de 1794 (RAH-9/3921/85), 3 de enero de 1795 (RAH9/3921/86), 7 de marzo de 1795 (RAH-9/3921/87), 21 de marzo de 1795 (RAH-9/3921/88), 8 de diciembre de 1795 (RAH-9/3921/90), 19 de marzo de 1796 (RAH-9/3921/92), 23 de diciembre de 1800 (RAH9/3921/93), 30 de mayo de 1801 (RAH-9/3921/94) y 28 de noviembre de 1801 (RAH-9/3921/95), con que termina la correspondencia entre ambos, una vez más aludiendo aunque de forma tangencial a Segobriga. La relación de todos los documentos de Juan Antonio Fernández que conserva la Real Academia de la Historia, incluyendo su correspondencia, se encuentra en J. M. Abascal, R. Cebrián, Los viajes... (op. cit. en nota 5) 204-209.

${ }^{60}$ Juan Antonio Fernández, Aqüeducto Caesaraugustano. Memorias y apuntaciones para escribir de este antiguo monumento. Por D. Juan Antonio Fernández, Archivero General de su Magestad del Orden de Santiago e individuo de la Real Academia de la Historia (Zaragoza 1804). Archivo de la Universidad de Oviedo, ms. 168. La obra ha sido editada y comentada con rigor en A. Vázquez de la Cueva, I. González Tascón, "El abastecimiento de agua romano a Caesaraugusta", Anas 1 (1988) 35-66; I. González Tascón, A Vázquez de la Cueva, J. L. Ramírez Sádaba, El acueducto romano de Caesaraugusta según el manuscrito de Juan Antonio Fernández (1752-1814) (Madrid 1994). 
que puso a su disposición las piezas, daría a Juan Antonio Fernández una nueva oportunidad para bucear en los estudios epigráficos, de convertirse de nuevo en epigrafista accidental, una tarea que concluyó con la redacción de una memoria editada en $1994^{61}$ y con la que Fernández se despediría definitivamente de los estudios sobre la antigüedad.

Si tuvo intención de continuar con los estudios epigráficos, no lo sabemos. Su regreso definitivo a Tudela en $1808^{62}$, y una Guerra de la Independencia por medio, le llevaron a la tumba el 23 de junio de 1814 sin ver cumplido el sueño de publicar sus estudios sobre Cabeza del Griego. No obstante, sus manuscritos ${ }^{63}$, su rico archivo, su biblioteca e incluso el monetario que había formado a lo largo de su vida, tenían un interés científico innegable que la Real Academia de la Historia supo valorar desde un primer momento.

Sabedora de ello, Francisca Uxaravi, la viuda de Juan Antonio Fernández y quien le había acompañado en sus estancias en Uclés y Zaragoza, instó en 1820 la realización de un censo de los documentos y del monetario de su difunto marido, que fue convenientemente tasado para ofrecerlo en venta a la Academia. Los responsables de esa tasación por parte de la familia fueron Nicolás María Sinlanas ${ }^{64}$, en nombre de la ciudad de Tudela, y Jorge de Uxaravi ${ }^{65}$, cuñado del fallecido ${ }^{66}$. Esa documentación fue remitida a Martín Fernández de Navarrete el 10 de enero de 1821, pero el precio solicitado, 20.000 reales de vellón en dinero efectivo por

${ }^{61}$ Cf. nota 60.

${ }^{62}$ D. Clemencín, Noticia de la Real Academia de la Historia o Resumen de sus Actas desde el año de 1821 hasta concluir el de 1831, leido en sus Juntas del mes de marzo de 1832 (Madrid 1832).

${ }^{63}$ Los inventarios de sus manuscritos de 1818 y 1834, así como el de su biblioteca, pueden verse en los trabajos de F. Mikelarena Peña citados en la nota 5. Esas relaciones incluyen algunos textos sobre $\mathrm{Se}$ gobriga. Cf. por ejemplo F. Mikelarena Peña, "Los manuscritos de la biblioteca...", op. cit. en nota 5, 284 y 294-295, n 53 (Escabazion de la Caveza llamada del Griego. Colección de todos sus descubrimientos y antigüedades y memorias de la ciudad de Segobriga), $\mathrm{n}^{\circ} 592$ (Noticias de la Escabacion del territorio de Cabeza del Griego) y n 595 (Escabacion del término de Cabeza del Griego). Cf. también M. Á. Colomo de Granda, M. . P. Jiménez Zamora, "Juan Antonio Fernández...”, op. cit. en nota 5. A esas colecciones hay que añadir los fondos que alberga la Biblioteca Nacional: Correspondencia de Juan Antonio Fernández, archivero general de la Orden de Santiago (B.N.E. Mss./17483, procedente de la colección de Pascual de Gayangos); Inscripciones nuevamente descubiertas en la villa de Santa Cara en el Reino de Navarra, la primera, en una columna grande de un zaguán, y las siguientes, en una piedra del palacio de dicha villa (B.N.E. Mss./10343, hojas 239r.-240r.); Monetario y manuscritos hallados en poder del anticuario Juan Antonio Fernández, al ocurrir su muerte en Tudela (B.N.E. Mss/18633/91); Cartas de Juan Antonio Fernández y de Félix Guitarte a Tomás López, sobre Tudela [fechadas en Tudela y Zaragoza, entre junio y octubre de 1784] (B.N.E. Mss./12978/10).

${ }^{64}$ No Siulanas como se cita en algunas ocasiones. Su firma autógrafa en el manuscrito RAH9-GN-1821-1828-1(1) es concluyente. Allí aparece como “tutor y tasador de D. Lázaro Fernández".

${ }^{65}$ No Urazavi, como se le cita en el catálogo de la Real Academia de la Historia (cf. F. Martín Escudero et alii, Real Academia de la Historia. Archivo del Gabinete del Numario. Catálogo e índices [Madrid 2004] 189). Era cuñado de Juan Antonio Fernández.

${ }^{66}$ Ms. RAH-9/GN/1821/1828/1(1): Tasación de los manuscritos y monedas que dejó a su muerte Juan Antonio Fernández, con descripción de los mismos. Tudela, 1 de enero de 1821. 
los manuscritos y otro tanto por las monedas ${ }^{67}$, debió de impedir un acuerdo inmediato. A la vista del informe interno elaborado por el académico Francisco Antonio González ${ }^{68}$, en la sesión del 2 de febrero de 1821 se comisionó a Fernández de Navarrete para pedir a la familia que enviaran los documentos y el monetario y que, a la vista de lo recibido, la Academia haría un juicio de valor más adecuado.

Las negociaciones se prolongarían aún durante unos años pero, finalmente, el 7 de agosto de 1828, Nicolás María Sinlanas informó a la Academia que los herederos de Juan Antonio Fernández ya tenían embalados los manuscritos y el monetario del difunto para enviarlos a Madrid, de manera que fuera posible tomar una decisión sobre el valor de las piezas y llegar a un acuerdo para formalizar la venta $^{69}$. Este cambio de actitud aceleró los trámites y una gran parte de los manuscritos y del monetario pasarían por venta a propiedad de la Academia, como relataría Diego Clemencín en su informe de gestión del período 1821-1834:

La Academia... practicó diligencias para que no se perdieran ni oscureciesen los manuscritos que dejó don Juan Antonio Fernández, archivero que fue de la orden de Santiago; en los cuales puede creerse que se habrán salvado noticias importantes, relativas a dicha orden, cuyo archivo general fue saqueado y destruido de resultas de la funesta batalla de Uclés en enero de mil ochocientos y nueve ${ }^{70}$.

Así fue como la Real Academia de la Historia se convirtió en depositaria de una gran parte del legado de Juan Antonio Fernández, lo que permitió reunir en un mismo archivo sus documentos, los informes de Antonio Tavira y la correspondencia oficial generada por aquellas lejanas excavaciones en Cabeza del Griego (Segobriga) en el invierno de 1789-1790. Por entonces, durante unos meses, el de Tudela demostró que no sólo entendía de archivos sino que tenía formación suficiente para ser también un buen epigrafista accidental.

${ }^{67}$ Oficio de Nicolás María Sinlanas a Martín Fernández de Navarrete. Tudela 10 de enero de 1821. Se conserva en la Real Academia de la Historia, signatura RAH-9/GN/1821-1828/1(2).

${ }^{68} \mathrm{El}$ informe daba a entender que un simple listado de monedas no servía para saber de su valor real, pero advertía a la Academia del interés que podía tener la serie de piezas visigodas. El informante también era cauto sobre el valor que pudieran tener unos manuscritos que no estaban adecuadamente descritos. Cf. Francisco Antonio González, Informe sobre el catálogo del monetario y manuscritos dejados a su muerte por el académico correspondiente Juan Antonio Fernández. RAH-9/GN/1821-1828/1(3), con fecha de 31 de enero de 1821 .

${ }^{69}$ Oficio de Nicolás María Sinlanas a Martín Fernández de Navarrete como secretario de la Academia. Tudela, 7 de agosto de 1828. RAH-9/GN/1821-1828/1(4): "los herederos del difunto antiquario Fernández buelben otra vez a incomodar la atencion de V. diciendole que tienen encajonados los libros y monetario y se allan decicidos a mandarlos a esa siempre que V. pueda asegurarnos que acomodando parte o el todo a la R(ea)l Academia, sera efectivo su pago...". Al pie de la carta: "Acad(emi)a de 16 de agosto de 1828: Sírvase de contestar el Sr. Director que los herederos pueden enviar los libros y monedas si gustan, y que con su vista la Acad(emi)a resolverá lo que tenga por conveniente".

${ }^{70}$ D. Clemencín, (op. cit. en nota 62) 14. 